\title{
Breast Swelling
}

National Cancer Institute

\section{Source}

National Cancer Institute. Breast Swelling. NCI Thesaurus. Code C34437.

Enlargement of the breast due to increased fluid volume. 